\title{
GROWTH ESTIMATES FOR SOLUTIONS OF NONLINEAR SECOND-ORDER DIFFERENCE EQUATIONS
}

\author{
STEVO STEVIĆ
}

(Received 17 April, 2004; revised 1 October, 2004)

\begin{abstract}
We prove several growth theorems for solutions of certain nonlinear second-order difference equations.
\end{abstract}

\section{Introduction}

In this paper we study the asymptotic behaviour of the second-order nonlinear difference equation:

$$
\Delta\left(c_{n-1} \Delta x_{n-1}\right)=f\left(n, x_{n}, \Delta x_{n-1}\right)+g\left(n, x_{n}, \Delta x_{n-1}\right), \quad n \in \mathbb{N},
$$

where $f$ and $g$ are real functions satisfying some additional conditions which we explain later. Our results are natural extensions and generalisations of those in [9] and are also closely related to those in [4] and [8], where the growth of the solutions of the corresponding homogeneous difference equation was investigated. This equation models, for example, the amplitude of oscillation of the weights on a discretely weighted vibrating string.

Similar equations have been investigated by many authors, see, for example [1, Chapter 6.15]. Our motivation here stems from [1, Problem 6.24.40]. According to [1] the following result holds.

THEOREM A. Consider the equation

$$
\Delta\left(c_{n-1} \Delta x_{n-1}\right)=d_{n} f\left(n, x_{n}, \Delta x_{n-1}\right)+g\left(n, x_{n}, \Delta x_{n-1}\right),
$$

where

\footnotetext{
${ }^{1}$ Mathematical Institute of Serbian Academy of Science, Knez Mihailova 35/I, 11000 Beograd, Serbia: e-mail: sstevic@ptt.yu and sstevo@matf.bg.ac.yu.

(C) Australian Mathematical Society 2005, Serial-fee code 1446-1811/05
} 
(a) $c_{n}>0, n \geq 0$ and $\sum_{i=1}^{\infty} 1 / c_{i-1}=\infty$;

(b) $|g(n, u, v)| \leq g_{n}$, where $g_{n}$ is a real sequence such that $\sum_{t=1}^{\infty} g_{n}<\infty$;

(c) $d_{n}$ is a real sequence such that $\sum_{j=1}^{+\infty}\left|d_{j}\right| \sum_{i=2}^{j} 1 / c_{i-1}<\infty$;

(d) $f$ is a real function such that $|f(n, u, v)| \leq L|u|, u, v \in \mathbb{R}, n \in \mathbb{N}$ for some $L>0$.

Then $x_{n}=\mathscr{O}\left(\sum_{i=2}^{n} 1 / c_{t-1}\right)$ as $n \rightarrow+\infty$.

We want to say that the following, slightly different, equation

$$
\Delta\left(c_{n} \Delta x_{n}\right)=d_{n} f\left(n, x_{n}, \Delta x_{n}\right)+g\left(n, x_{n}, \Delta x_{n}\right)
$$

is considered in [1]. However, it is easy to see that under the same conditions as in Theorem A the same asymptotic formula holds (see also the proof of Lemma 2.4). Theorems 3.1, 3.5 and 3.6 generalise and extend Theorem A in many directions. In Section 2 we quote three auxiliary results which we apply in the third section. In Section 3 we study the asymptotic behaviour of the solutions of the second-order nonlinear difference equation (1.1).

\section{Auxiliary results}

In order to investigate the growth of the solution $x_{n}$, we first need two auxiliary results which are discrete variants of Bellman-Gronwall's lemma. Applications and further generalisations of this lemma can be found, for example, in [2-10].

LEMMA 2.1 ([6, page 112]). If $x_{n}, b_{n}, c_{n} \geq 0$ and

$$
x_{n} \leq a_{n}+b_{n} \sum_{i=1}^{n-1} c_{i} x_{i}, \quad n \in \mathbb{N},
$$

then

$$
x_{n} \leq a_{n}+b_{n} \sum_{i=1}^{n-1} a_{l} c_{1} e^{\sum_{j=i+1}^{n-1} b, c_{j}}, \quad n \in \mathbb{N} .
$$

From Lemma 2.1 and the inequality $1+x \leq e^{x}$, for $x \geq 0$, it is easy to get the following corollary.

COROLLARY 2.2 ([3, page 198]). If $x_{n}, c_{n} \geq 0, c$ is a positive constant and

$$
x_{n} \leq c+\sum_{i=1}^{n-1} c_{i} x_{i}, \quad n \in \mathbb{N},
$$

then $x_{n} \leq c e^{\sum_{i=1}^{n-1} r_{1}}, n \in \mathbb{N}$. 
The following lemma is a special case of [10, Theorem 4].

LEMMA 2.3. If $x_{n}, c_{n} \geq 0, c$ is a positive constant, $p \in[0,1)$ and

$$
x_{n} \leq c+\sum_{i=1}^{n-1} c_{i} x_{i}^{p}, \quad n \in \mathbb{N}
$$

then

$$
x_{n} \leq\left(c^{1-p}+(1-p) \sum_{i=1}^{n-1} c_{i}\right)^{1 /(1-p)} \quad, \quad n \in \mathbb{N}
$$

The next result can be considered as the main technical part of this paper.

LEMMA 2.4. Consider (1.1) where

(a) $c_{n}>0, n \geq 0$;

(b) $f$ and $g$ are real functions; and

(c) $|g(n, u, v)| \leq g_{n}$, where $g_{n}$ is an arbitrary real sequence.

Then the following inequalities hold:

$$
\left|\Delta x_{n-1}\right| \leq \frac{1}{c_{n-1}}\left(\left|y_{0}\right|+\sum_{i=1}^{n-1} g_{i}+\sum_{i=1}^{n-1}\left|f\left(i, x_{i}, \Delta x_{i-1}\right)\right|\right)
$$

and

$$
\frac{\left|x_{n}\right|}{\sum_{t=2}^{n} 1 / c_{i-1}} \leq c_{1}\left|x_{1}\right|+\left|y_{0}\right|+\sum_{i=1}^{n-1} g_{i}+\sum_{i=1}^{n-1}\left|f\left(i, x_{i}, \Delta x_{i-1}\right)\right| .
$$

PROOF. Let $y_{n}=c_{n}\left(x_{n+1}-x_{n}\right)$. Then from (1.1) we have

$$
y_{n}-y_{n-1}=f\left(n, x_{n}, \Delta x_{n-1}\right)+g\left(n, x_{n}, \Delta x_{n-1}\right), \quad n \in \mathbb{N} .
$$

Summing (2.3) from 1 to $n-1$, we obtain

$$
x_{n}-x_{n-1}=\frac{1}{c_{n-1}}\left(y_{0}+\sum_{i=1}^{n-1}\left(f\left(i, x_{i}, \Delta x_{i-1}\right)+g\left(i, x_{i}, \Delta x_{i-1}\right)\right)\right) \text {. }
$$

From this and condition (c) we get

$$
\left|\Delta x_{n-1}\right| \leq \frac{1}{c_{n-1}}\left(\left|y_{0}\right|+\sum_{i=1}^{n-1} g_{i}+\sum_{i=1}^{n-1}\left|f\left(i, x_{i}, \Delta x_{i-1}\right)\right|\right) .
$$

Summing (2.4) from 2 to $n$, we have

$$
x_{n}=x_{1}+y_{0} \sum_{i=2}^{n} \frac{1}{c_{i-1}}+\sum_{i=2}^{n} \frac{1}{c_{i-1}}\left(\sum_{j=1}^{i-1}\left(f\left(j, x_{j}, \Delta x_{j-1}\right)+g\left(j, x_{j}, \Delta x_{j-1}\right)\right)\right) \text {, }
$$


which yields

$$
\left|x_{n}\right| \leq\left|x_{1}\right|+\left|y_{0}\right| \sum_{i=2}^{n} \frac{1}{c_{i-1}}+\sum_{i=2}^{n} \frac{1}{c_{i-1}} \sum_{j=1}^{i-1} g_{j}+\sum_{i=2}^{n} \frac{1}{c_{i-1}} \sum_{j=1}^{i-1}\left|f\left(j, x_{j}, \Delta x_{j-1}\right)\right|
$$

and consequently

$$
\frac{\left|x_{n}\right|}{\sum_{i=2}^{n} 1 / c_{i-1}} \leq c_{1}\left|x_{1}\right|+\left|y_{0}\right|+\sum_{i=1}^{n-1} g_{i}+\sum_{i=1}^{n-1}\left|f\left(i, x_{i}, \Delta x_{i-1}\right)\right|
$$

as desired.

\section{The main results}

In this section we study the asymptotic behaviour of the solutions of the secondorder nonlinear difference equation (1.1).

THEOREM 3.1. Consider (1.1) where

(a) $c_{n}>0, n \geq 0$;

(b) $|g(n, u, v)| \leq g_{n}$, where $g_{n}$ is an arbitrary real sequence; and

(c) $f$ is a real function such that $|f(n, u, v)| \leq\left|d_{n}\right||u|^{\alpha}, u, v \in \mathbb{R}, n \in \mathbb{N}$, for some $\alpha \in[0,1]$ and a real sequence $d_{n}$.

Then the following asymptotic formula holds:

$$
x_{n}=\mathscr{O}\left(a_{n}+\sum_{i=2}^{n} \frac{1}{c_{i-1}} \sum_{j=1}^{n-1} a_{j}\left|d_{j}\right| e^{\sum_{k=j+1}^{n-1}\left|d_{k}\right| \sum_{i=2}^{k} 1 / c_{i-1}}\right) \text { as } n \rightarrow+\infty,
$$

where $a_{n}=\sum_{t=2}^{n}\left(1+\sum_{j=1}^{i-1} g_{\jmath}\right) / c_{i-1}$.

Proof. Let $y_{n}=c_{n}\left(x_{n+1}-x_{n}\right)$. From inequality (2.5) in Lemma 2.4 and by condition (c) we have

$$
\left|x_{n}\right| \leq\left|x_{1}\right|+\left|y_{0}\right| \sum_{i=2}^{n} \frac{1}{c_{i-1}}+\sum_{i=2}^{n} \frac{1}{c_{i-1}} \sum_{j=1}^{i-1} g_{j}+\sum_{i=2}^{n} \frac{1}{c_{i-1}} \sum_{j=1}^{i-1}\left|d_{j}\right|\left|x_{j}\right|^{\alpha} .
$$

From this and by the well-known inequality $|x|^{\alpha} \leq 1+|x|, x \in \mathbb{R}, \alpha \in[0,1]$, we have

$$
\begin{aligned}
\left|x_{n}\right|+1 & \leq\left|x_{1}\right|+1+\left|y_{0}\right| \sum_{i=2}^{n} \frac{1}{c_{t-1}}+\sum_{i=2}^{n} \frac{1}{c_{i-1}} \sum_{j=1}^{i-1} g_{j}+\sum_{i=2}^{n} \frac{1}{c_{i-1}} \sum_{j=1}^{i-1}\left|d_{j}\right|\left(\left|x_{j}\right|+1\right) \\
& \leq\left|x_{1}\right|+1+\left|y_{0}\right| \sum_{i=2}^{n} \frac{1}{c_{i-1}}+\sum_{i=2}^{n} \frac{1}{c_{i-1}} \sum_{j=1}^{i-1} g_{j}+\sum_{i=2}^{n} \frac{1}{c_{i-1}} \sum_{j=1}^{n-1}\left|d_{j}\right|\left(\left|x_{j}\right|+1\right) .
\end{aligned}
$$


Let $\hat{a}_{n}=\left|x_{1}\right|+1+\left|y_{0}\right| \sum_{i=2}^{n}\left(1 / c_{i-1}\right)+\sum_{i=2}^{n}\left(\sum_{j=1}^{t-1} g_{j}\right) / c_{i-1}$, then by Lemma 2.1,

$$
\left|x_{n}\right|+1 \leq \hat{a}_{n}+\sum_{i=2}^{n} \frac{1}{c_{i-1}} \sum_{j=1}^{n-1} \hat{a}_{j}\left|d_{j}\right| e^{\sum_{k=j+1}^{n-1}\left|d_{k}\right| \sum_{i=2}^{k} 1 / r_{i-1}}
$$

Since $a_{n}$ is increasing we have

$$
1 \leq a_{1}\left(\left[\frac{1}{a_{1}}\right]+1\right) \leq a_{n}\left(\left[\frac{1}{a_{1}}\right]+1\right), \quad n \geq 1 .
$$

On the other hand, we have $\left|\hat{a}_{n}\right| \leq \max \left\{\left|x_{1}\right|+1,\left|y_{0}\right|, 1\right\}\left(1+a_{n}\right)$, for $n \geq 1$, which implies

$$
\left|\hat{a}_{n}\right| \leq \max \left\{\left|x_{1}\right|+1,\left|y_{0}\right|, 1\right\}\left(\left[\frac{1}{a_{1}}\right]+2\right) a_{n}, \quad n \geq 1 .
$$

From this and (3.1) the result follows.

\section{COROLlaRY 3.2. Consider (1.1) where}

(a) $c_{n}>0, n \geq 0$ and $\sum_{i=1}^{\infty} 1 / c_{i-1}=\infty$;

(b) $|g(n, u, v)| \leq g_{n}$, where $g_{n}$ is an arbitrary real sequence;

(c) $d_{n}$ is a real sequence such that $\sum_{j=1}^{+\infty}\left|d_{j}\right| \sum_{i=2}^{j} 1 / c_{i-1}<\infty$; and

(d) $f$ is a real function such that $|f(n, u, v)| \leq\left|d_{n}\right||u|^{\alpha}, u, v \in \mathbb{R}, n \in \mathbb{N}$, for some $\alpha \in[0,1]$.

Then the following asymptotic formula holds:

$$
x_{n}=\mathscr{O}\left(\sum_{i=2}^{n} \frac{1}{c_{i-1}}\left(\sum_{i=2}^{n} \frac{1}{c_{i-1}}\left(1+\sum_{j=1}^{i-1} g_{i}\right)\right)\right) \text { as } n \rightarrow+\infty .
$$

PROOF. Let $a_{n}$ and $\hat{a}_{n}$ be as in Theorem 3.1. Dividing (3.1) by $a_{n} \sum_{i=2}^{n} 1 / c_{i-1}$ and using conditions (a) and (c) we obtain that there are positive constants $C_{1}, C_{2}, C_{3}$ and $C_{4}$ such that

$$
\frac{\left|x_{n}\right|+1}{a_{n} \sum_{i=2}^{n} 1 / c_{i-1}} \leq C_{1}+C_{2} \sum_{j=1}^{n-1}\left|d_{j}\right| \leq C_{3}+C_{4} \sum_{j=1}^{n-1}\left|d_{j}\right| \sum_{i=2}^{j} \frac{1}{c_{t-1}}<\infty
$$

as desired.

COROLlary 3.3. Consider (1.1) where

(a) $c_{n}>0, n \geq 0$ and $\sum_{i=1}^{\infty} 1 / c_{i-1}=\infty$;

(b) $|g(n, u, v)| \leq g_{n}$, where $g_{n}$ is a real sequence such that $\sum_{i=1}^{\infty} g_{n}<\infty$; 
(c) $d_{n}$ is a real sequence such that $\sum_{j=1}^{+\infty}\left|d_{j}\right| \sum_{t=2}^{j} 1 / c_{t-1}<\infty$; and

(d) $f$ is a real function such that, $|f(n, u, v)| \leq\left|d_{n}\right||u|^{\alpha}, u, v \in \mathbb{R}, n \in \mathbb{N}$, for some $\alpha \in[0,1]$.

Then $x_{n}=\mathscr{O}\left(\sum_{t=2}^{n} 1 / c_{i-1}\right)$ as $n \rightarrow+\infty$.

PROOF. Since $\sum_{t=1}^{\infty} g_{n}<\infty$ we have $a_{n} \asymp \sum_{i=2}^{n} 1 / c_{i-1}$. From this and (3.1) we obtain that there are positive constants $C_{1}$ and $C_{2}$ such that

$$
\begin{aligned}
\left|x_{n}\right|+1 & \leq \sum_{i=2}^{n} \frac{1}{c_{i-1}}\left(C_{1}+C_{2} \sum_{j=1}^{n-1}\left|d_{j}\right| \sum_{i=2}^{j} \frac{1}{c_{i-1}} e^{\sum_{k=j+1}^{n-1}\left|d_{k}\right| \sum_{i=2}^{k} 1 / c_{i-1}}\right) \\
& \leq \sum_{i=2}^{n} \frac{1}{c_{i-1}}\left(C_{1}+C_{3} e^{\sum_{j=1}^{n-1}\left|d_{j}\right| \sum_{i=2}^{\prime} 1 / c_{i-1}}\right) \leq C_{4} \sum_{i=2}^{n} \frac{1}{c_{t-1}}
\end{aligned}
$$

as desired.

REMARK. Note that Corollary 3.3 in the case $\alpha=1$ reduces to Theorem A ([1, Problem 6.24.40]).

EXAMPLE 1. Consider the equation

$$
\Delta\left(\sqrt{n+1} \Delta x_{n-1}\right)=\frac{\sqrt{\left|x_{n}\right|}}{n^{2}\left(1+\left(\Delta x_{n-1}\right)^{2}\right)}+\frac{1}{n^{3}+x_{n}^{2}}, \quad n \in \mathbb{N} .
$$

Here we have $c_{n}=\sqrt{n+2}$ and $d_{n}=1 / n^{2}$. Since $\sum_{t=2}^{\infty} 1 / \sqrt{i+1}=\infty$, condition (a) in Corollary 3.3 is satisfied. Condition (b) follows from the following inequality:

$$
\sum_{n=1}^{\infty} \frac{1}{n^{3}+x_{n}^{2}}<\sum_{n=1}^{\infty} \frac{1}{n^{3}}<\infty
$$

Using the asymptotics

$$
\sum_{i=2}^{j} \frac{1}{\sqrt{i+1}} \sim \int_{2}^{j} \frac{d x}{\sqrt{x+1}} \sim 2 \sqrt{j}
$$

we get

$$
\sum_{j=1}^{+\infty}\left|d_{j}\right| \sum_{i=2}^{j} \frac{1}{c_{i-1}} \asymp \sum_{j=1}^{+\infty} \frac{1}{j^{3 / 2}}<\infty,
$$

that is, condition (c) is satisfied. Condition (d) holds for $\alpha=1 / 2$, since

$$
\left|f\left(n, x_{n}, \Delta x_{n-1}\right)\right|=\frac{\sqrt{\left|x_{n}\right|}}{n^{2}\left(1+\left(\Delta x_{n-1}\right)^{2}\right)} \leq \frac{1}{n^{2}}\left|x_{n}\right|^{1 / 2} .
$$

Hence by Corollary 3.3 we have $x_{n}=\mathscr{O}\left(\sum_{t=2}^{n} 1 / \sqrt{i+1}\right)=\mathscr{O}(\sqrt{n})$. 
COROLlary 3.4. Consider (1.1) where

(a) $c_{n}>\delta>0, n \geq 0$;

(b) $|g(n, u, v)| \leq g_{n}$, where $g_{n}$ is a real sequence such that $\sum_{i=1}^{\infty} g_{n}<\infty$;

(c) $d_{n}$ is a real sequence such that $\sum_{j=1}^{+\infty}\left|d_{j}\right| \sum_{i=2}^{J} 1 / c_{i-1}<\infty$; and

(d) $f$ is a real function such that $|f(n, u, v)| \leq\left|d_{n}\right||u|^{\alpha}, u, v \in \mathbb{R}, n \in \mathbb{N}$, for some $\alpha \in[0,1]$.

Then $x_{n}=\mathscr{O}(n)$ as $n \rightarrow+\infty$.

PROOF. Since in this case $\sum_{i=2}^{n} 1 / c_{i-1}<(n-1) / \delta$, we obtain the result by Corollary 3.3 .

THEOREM 3.5. Consider (1.1) where

(a) $c_{n}>0, n \geq 0$ and $\sum_{i=1}^{\infty} 1 / c_{i-1}=\infty$;

(b) $|g(n, u, v)| \leq g_{n}$, where $g_{n}$ is a real sequence such that $\sum_{t=1}^{\infty} g_{n}<\infty$;

(c) $d_{n}$ is a real sequence such that, for some $\alpha \in[0,1)$,

$$
\sum_{i=1}^{+\infty}\left|d_{i}\right|\left(\sum_{j=2}^{i} \frac{1}{c_{j-1}}\right)^{\alpha}<\infty ; \quad \text { and }
$$

(d) $f$ is a real function such that $|f(n, u, v)| \leq\left|d_{n}\right||u|^{\alpha}, u, v \in \mathbb{R}, n \in \mathbb{N}$.

Then the following asymptotic formula holds:

$$
x_{n}=\mathscr{O}\left(\sum_{i=2}^{n} \frac{1}{c_{i-1}}\right) \text { as } n \rightarrow+\infty,
$$

for every solution $x_{n}$ of (1.1) and $\lim _{n \rightarrow+\infty} c_{n-1}\left(x_{n}-x_{n-1}\right)$ is finite.

Proof. From (2.5), conditions (b) and (c) and some simple calculations we get

$$
\begin{aligned}
\left|x_{n}\right| & \leq\left|x_{1}\right|+\left|y_{0}\right| \sum_{i=2}^{n} \frac{1}{c_{i-1}}+\sum_{i=2}^{n} \frac{1}{c_{i-1}} \sum_{j=1}^{i-1} g_{j}+\sum_{i=2}^{n} \frac{1}{c_{i-1}} \sum_{j=1}^{1-1}\left|d_{j}\right|\left|x_{j}\right|^{\alpha} \\
& \leq\left|x_{1}\right|+C \sum_{i=2}^{n} \frac{1}{c_{i-1}}+\sum_{i=2}^{n} \frac{1}{c_{i-1}} \sum_{j=1}^{i-1}\left|d_{j}\right|\left|x_{j}\right|^{\alpha} \\
& =\left|x_{1}\right|+C \sum_{i=2}^{n} \frac{1}{c_{i-1}}+\sum_{j=1}^{n-1}\left|d_{j}\right|\left|x_{j}\right|^{\alpha} \sum_{i=j+1}^{n} \frac{1}{c_{i-1}} \\
& \leq\left|x_{1}\right|+\sum_{i=2}^{n} \frac{1}{c_{i-1}}\left(C+\sum_{j=1}^{n-1}\left|d_{j}\right|\left|x_{j}\right|^{\alpha}\right)
\end{aligned}
$$


where $C=\left|y_{0}\right|+\sum_{n=1}^{\infty} g_{n}$.

From (3.2) we have

$$
\frac{\left|x_{n}\right|}{\sum_{i=2}^{n} 1 / c_{i-1}} \leq C_{1}\left|x_{1}\right|+C+\sum_{j=1}^{n-1}\left(\sum_{i=2}^{j} \frac{1}{c_{i-1}}\right)^{\alpha}\left|d_{j}\right| \frac{\left|x_{j}\right|^{\alpha}}{\left(\sum_{i=2}^{j} 1 / c_{t-1}\right)^{\alpha}} .
$$

For $\alpha \in[0,1)$, by Lemma 2.3 we get

$$
\frac{\left|x_{n}\right|}{\sum_{i=2}^{n} 1 / c_{i-1}} \leq\left(\left(C_{1}\left|x_{1}\right|+C\right)^{1-\alpha}+(1-\alpha) \sum_{i=1}^{n-1}\left(\sum_{j=2}^{i} \frac{1}{c_{j-1}}\right)^{\alpha}\left|d_{i}\right|\right)^{1 /(1-\alpha)}<\infty,
$$

in view of condition (c), from which the first part of the theorem follows.

From the above we know that there exists $M>0$, such that $\left|x_{n}\right| \leq M \sum_{i=2}^{n} 1 / c_{i-1}$, for every $n \in \mathbb{N}$. Summing (2.3) from $n+1$ to $n+p$, we obtain

$$
y_{n+p}-y_{n}=\sum_{i=n+1}^{n+p}\left(f\left(i, x_{i}, \Delta x_{i-1}\right)+g\left(i, x_{i}, \Delta x_{i-1}\right)\right) .
$$

Hence

$$
\left|y_{n+p}-y_{n}\right| \leq \sum_{i=n+1}^{n+p} g_{i}+\sum_{i=n+1}^{n+p}\left|d_{i}\right|\left|x_{t}\right|^{\alpha} \leq \sum_{i=n+1}^{n+p} g_{l}+M^{\alpha} \sum_{i=n+1}^{n+p}\left(\sum_{j=2}^{i} \frac{1}{c_{j-1}}\right)^{\alpha}\left|d_{l}\right| .
$$

By the conditions of the theorem and Cauchy's criteria we obtain the result.

EXAMPLE 2. Consider the equation

$$
\Delta\left(\sqrt{n+1} \Delta x_{n-1}\right)=\frac{\sqrt{\left|x_{n}\right|}}{n^{3 / 2}\left(1+\left(\Delta x_{n-1}\right)^{2}\right)}+\frac{1}{n^{2}+\left(\Delta x_{n-1}\right)^{2}}, \quad n \in \mathbb{N} .
$$

Here $c_{n}=\sqrt{n+2}, d_{n}=1 / n^{3 / 2}$ and $\alpha=1 / 2$. As in Example 1 it can be shown that the conditions in Theorem 3.5 are satisfied. Therefore

$$
x_{n}=\mathscr{O}\left(\sum_{i=2}^{n} \frac{1}{\sqrt{i+1}}\right)=\mathscr{O}(\sqrt{n})
$$

and the $\operatorname{limit}_{n \rightarrow \infty} \sqrt{n+1}\left(x_{n}-x_{n-1}\right)$ is finite.

Note that this equation does not satisfy condition (c) in Corollary 3.3, since

$$
\sum_{j=1}^{+\infty}\left|d_{j}\right| \sum_{i=2}^{j} \frac{1}{c_{i-1}} \asymp \sum_{j=1}^{+\infty} \frac{1}{j}=\infty
$$


The following result also includes the case $\alpha=1$, moreover, we impose a condition on the rate of the growth of the function $f$ which also includes the difference $\Delta x_{n-1}$.

\section{THEOREM 3.6. Consider (1.1) where}

(a) $c_{n} \geq \delta>0, n \geq 0$;

(b) $|g(n, u, v)| \leq g_{n}$, where $g_{n}$ is a real sequence such that $\sum_{i=1}^{\infty} g_{n}<\infty$;

(c) $f$ is a real function such that $|f(n, u, v)| \leq d_{n}|u|^{\alpha}+f_{n}|v|^{\beta}, u, v \in \mathbb{R}, n \in \mathbb{N}$, for some $\alpha, \beta \in[0,1]$ and real sequences $d_{n}$ and $f_{n}$; and

(d) the series $\sum_{n=1}^{\infty}\left|d_{i}\right|\left(\sum_{j=2}^{l} 1 / c_{j-1}\right)^{\alpha}$ and $\sum_{n=1}^{\infty} f_{n}$ are convergent.

Then $x_{n}=\mathscr{O}\left(\sum_{i=2}^{n} 1 / c_{i-1}\right)$ as $n \rightarrow+\infty$, for every solution $x_{n}$ of $(1.1)$ and the limit $\lim _{n \rightarrow+\infty} c_{n-1}\left(x_{n}-x_{n-1}\right)$ is finite.

ProOF. Since the conditions of Lemma 2.4 are satisfied, summing inequalities (2.1) and (2.2), and using condition (a), we obtain

$$
\begin{aligned}
\left|\Delta x_{n-1}\right|+\frac{\left|x_{n}\right|}{\sum_{i=2}^{n} 1 / c_{i-1}} \leq & \frac{\left|y_{0}\right|}{\delta}+c_{1}\left|x_{1}\right|+\left|y_{0}\right| \\
& +\left(\frac{1}{\delta}+1\right)\left(\sum_{i=1}^{n-1} g_{i}+\sum_{i=1}^{n-1}\left|f\left(i, x_{i}, \Delta x_{i-1}\right)\right|\right) .
\end{aligned}
$$

hence by conditions (b) and (c) we get

$$
\begin{aligned}
\left|\Delta x_{n-1}\right|+\frac{\left|x_{n}\right|}{\sum_{i=2}^{n} 1 / c_{t-1}} & \leq C+C\left(\sum_{i=1}^{n-1} g_{i}+\sum_{i=1}^{n-1}\left(\left|d_{j}\right|\left|x_{\jmath}\right|^{\alpha}+\left|f_{j}\right|\left|\Delta x_{\jmath-1}\right|^{\beta}\right)\right) \\
& \leq C_{1}+C \sum_{i=1}^{n-1}\left(\left|d_{j}\right|\left|x_{j}\right|^{\alpha}+\left|f_{j} \| \Delta x_{\jmath}-1\right|^{\beta}\right),
\end{aligned}
$$

where $C_{1}=C\left(1+\sum_{n=1}^{\infty} g_{n}\right)$, or equivalently

$$
\begin{aligned}
& \left|\Delta x_{n-1}\right|+\frac{\left|x_{n}\right|}{\sum_{i=2}^{n} 1 / c_{i-1}} \\
& \leq C_{1}+C \sum_{j=1}^{n-1}\left(\left(\sum_{i=2}^{j} \frac{1}{c_{i-1}}\right)^{\alpha}\left|d_{j}\right| \frac{\left|x_{j}\right|^{\alpha}}{\left(\sum_{i=2}^{j} 1 / c_{i-1}\right)^{\alpha}}+\left|f_{j}\right|\left|\Delta x_{j-1}\right|^{\beta}\right) .
\end{aligned}
$$

Using the inequality $x^{t} \leq 1+x+y$, for $x, y \geq 0$ and $t \in(0,1]$, we get

$$
\begin{aligned}
& \left|\Delta x_{n-1}\right|+\frac{\left|x_{n}\right|}{\sum_{i=2}^{n} 1 / c_{i-1}} \\
& \leq C_{1}+C \sum_{j=1}^{n-1}\left(\left(\sum_{i=2}^{j} \frac{1}{c_{i-1}}\right)^{\alpha}\left|d_{j}\right|+\left|f_{j}\right|\right)\left(1+\frac{\left|x_{j}\right|}{\sum_{i=2}^{j} 1 / c_{i-1}}+\left|\Delta x_{j-1}\right|\right) .
\end{aligned}
$$


By Corollary 2.2 we have

$$
\begin{aligned}
1+\left|\Delta x_{n-1}\right| & +\frac{\left|x_{n}\right|}{\sum_{i=2}^{n} 1 / c_{i-1}} \\
& \leq\left(1+C_{1}\right) \exp \left(C\left(\sum_{j=1}^{n-1}\left(\sum_{i=2}^{j} \frac{1}{c_{i-1}}\right)^{\alpha}\left|d_{j}\right|+\left|f_{j}\right|\right)\right)<\infty,
\end{aligned}
$$

in view of condition (d), from which the first part of the theorem follows.

The rest of the proof is similar to the corresponding proof of Theorem 3.5 and will be omitted.

EXAMPLE 3. Consider the equation

$$
\Delta\left(\sqrt[3]{n+1} \Delta x_{n-1}\right)=\frac{\sqrt{\left|x_{n}\right|}+n^{1 / 4} \sqrt[4]{\Delta x_{n-1}}}{n^{3 / 2}\left(1+\left(\Delta x_{n-1}\right)^{2}\right)}+\frac{n}{2^{n}+\left|\Delta x_{n-1}\right|}, \quad n \in \mathbb{N} .
$$

Here $c_{n}=\sqrt[3]{n+2}, d_{n}=1 / n^{3 / 2}, f_{n}=1 / n^{5 / 4}, \alpha=1 / 2$ and $\beta=1 / 4$. It is easy to see that the conditions in Theorem 3.6 are satisfied. Thus $x_{n}=\mathscr{O}\left(\sum_{t=2}^{n} 1 / \sqrt[3]{i+1}\right)=$ $\mathscr{O}\left(n^{2 / 3}\right)$ and $\lim _{n \rightarrow \infty} \sqrt[3]{n+1}\left(x_{n}-x_{n-1}\right)$ is finite.

\section{References}

[1] R. P. Agarwal, Difference equations and inequalities, Pure Appl. Math. 228, 2nd ed. (Marcel Dekker, New York, 2000).

[2] F. S. De Blasi and J. Schinas, "On the stability of difference equations in Banach spaces", Analele stintificae ale Universitatii Al. Cuza, lasi Sectia la Matematica 20 (1974) 56-80.

[3] S. N. Elaydi, An introduction to difference equations (Springer, New York, 1996).

[4] E. Kurpinar and G. Sh. Guseinov, "The boundedness of solutions of the second-order difference equations", Indian J. Math. 37 (1995) 113-122.

[5] A. Mate and P. Nevai, "Sublinear perturbations of the differential equation $y^{(n)}=0$ and of the analogous difference equation", J. Differential Equations 53 (1984) 234-257.

[6] D. S. Mitrinović and J. E. Pečarić, Differential and integral inequalities (Naučna knjiga, Beograd, 1988).

[7] C. Papaschinopoulos, “On the summable manifold for discrete systems”, Math. Japonica 33 (1988) $457-468$.

[8] S. Stević, "Growth theorems for homogeneous second-order difference equations", ANZIAM J. 43 (2002) 559-566.

[9] S. Stević, "Asymptotic behaviour of second-order difference equations", ANZIAM J. 46 (2004) $157-170$.

[10] D. Willet and J. S. W. Wong, "On the discrete analogues of some generalizations of Gronwall's inequality", Monatsh. Math. 69 (1965) 362-367. 